\title{
A ZETA-FUNCTION ASSOCIATED WITH ZERO TERNARY FORMS ${ }^{1}$
}

\author{
MINKING EIE
}

ABSTRACT. Consider the zeta-function associated with zero ternary forms defined as

$$
\tilde{\xi}(t)=\sum_{x} \frac{1}{|\operatorname{det} x|^{t}} \quad(\operatorname{Re} t \geqslant 2),
$$

where $x$ runs over all $\mathrm{SL}_{3}(\mathbf{Z})$-inequivalent zero ternary forms. We shall approximate $\tilde{\xi}(t)$ by another zeta-function which we can compute explicitly. By the approximation, we see that $\tilde{\xi}(2)$ is very close to $2 \xi(2) \zeta(2)$ which gives the contribution of zero ternary forms to the dimension formula of Siegel's cusp forms of degree three (computing via Selberg Trace Formula) up to a constant multiple.

1. Introduction. For each pair of nonzero integers $s_{13}$ and $s_{2}$, we define $\Delta\left(s_{13}, s_{2}\right)$ to be the set of ternary forms

$$
\left[\begin{array}{ccc}
0 & 0 & s_{13} \\
0 & s_{2} & s_{23} \\
s_{13} & s_{23} & s_{3}
\end{array}\right]
$$

Let

$$
\mathscr{P}=\left\{U=\left[\begin{array}{lll}
1 & u & v \\
0 & 1 & u \\
0 & 0 & 1
\end{array}\right] \mid u, v, w \text { integers }\right\}
$$

$\mathscr{P}$ operates on $\Delta\left(s_{13}, s_{2}\right)$ by the action $S \rightarrow{ }^{t} U S U$. Let $\mu\left(s_{13}, s_{2}\right)$ be the number of inequivalent representatives of $\Delta\left(s_{13}, s_{2}\right)$ under the operation of $\mathscr{P}$. Consider the zeta function $\xi(t)$ defined as

$$
\xi(t)=\sum_{s_{2} \neq 0} \sum_{s_{13}=1}^{\infty} \frac{\mu\left(s_{13}, s_{2}\right)}{\left(s_{2} s_{13}^{2}\right)^{t}} .
$$

We shall prove

THEOREM A. For $\operatorname{Re} t \geqslant 2$, we have

$$
\xi(t)=\left\{\frac{5}{2}+\frac{3}{2}\left(\frac{2^{-2 t+1}+2^{-3 t}-2^{-5 t+2}}{1-2^{-3 t}}\right)\right\} \frac{\zeta(t) \zeta(2 t-1) \zeta(3 t-1)}{\zeta(3 t)},
$$

where $\zeta(t)$ is the Riemann zeta-function.

Received by the editors April 12, 1984 and, in revised form, September 26, 1984.

1980 Mathematics Subject Classification. Primary 10D20; Secondary 10A20.

${ }^{1}$ This work was supported by Academia Sinica and N.S.F. of Taiwan, Republic of China. 


\section{The special case $t=2$.}

LEMMA 1. Let $\delta\left(s_{13}, s_{2}\right)$ be the subset of $\Delta\left(s_{13}, s_{2}\right)$, defined by

$$
\left\{\begin{array}{l}
0 \leqslant s_{23}<\left(s_{13}, s_{2}\right)=\text { g.c.d. of } s_{13} \text { and } s_{2}, \\
0 \leqslant s_{3}<l\left(s_{23}\right)
\end{array}\right.
$$

where $l\left(s_{23}\right)$ is the least positive integer in the set

$$
G=\left\{2 k \cdot \frac{s_{23} s_{13}}{\left(s_{13}, s_{2}\right)}+k^{2} \cdot \frac{s_{13}^{2} s_{2}}{\left(s_{13}, s_{2}\right)^{2}}+2 n s_{13} \mid k, n \in \mathbf{Z}\right\}
$$

Then we have

(1) for each $S \in \Delta\left(s_{13}, s_{2}\right)$, there exists $U \in \mathscr{P}_{\text {such }}$ that ${ }^{t} U S U \in \delta\left(s_{13}, s_{2}\right)$; and

(2) if $S_{1}, S_{2} \in \delta\left(s_{13}, s_{2}\right), U \in \mathscr{P}$ and $S_{1}={ }^{t} U S_{2} U$, then $S_{1}=S_{2}$.

Proof. For

$$
U=\left[\begin{array}{ccc}
1 & m & n \\
0 & 1 & p \\
0 & 0 & 1
\end{array}\right] \text { and } S=\left[\begin{array}{ccc}
0 & 0 & s_{13} \\
0 & s_{2} & s_{23} \\
s_{13} & s_{23} & s_{3}
\end{array}\right]
$$

in $\mathscr{P}$ and $\Delta\left(s_{13}, s_{2}\right)$, respectively, we let

$$
{ }^{t} U S U=\left[\begin{array}{ccc}
0 & 0 & s_{13} \\
0 & s_{2} & s_{23}^{\prime} \\
s_{13} & s_{23}^{\prime} & s_{3}^{\prime}
\end{array}\right]
$$

Then a simple calculation shows

$$
\begin{aligned}
& s_{23}^{\prime}=s_{23}+m s_{13}+p s_{2}, \\
& s_{3}^{\prime}=s_{3}+2 p s_{23}+p^{2} s_{2}+2 n s_{13} .
\end{aligned}
$$

First we choose integers $m, p$ so that $0 \leqslant s_{23}^{\prime}<\left(s_{13}, s_{2}\right)$. Note that the integral solutions of the equation $m s_{13}+p s_{2}=0$ are given by

$$
p=\frac{k s_{13}}{\left(s_{13}, s_{2}\right)}, \quad m=\frac{-k s_{2}}{\left(s_{13}, s_{2}\right)}, \quad k \text { an integer. }
$$

Substituting the value of $p$ as above into $s_{3}^{\prime}$, we get

$$
s_{3}^{\prime}=s_{3}+2 k \cdot \frac{s_{23} s_{13}}{\left(s_{13}, s_{2}\right)}+k^{2} \cdot \frac{s_{13}^{2} s_{2}}{\left(s_{13}, s_{2}\right)^{2}}+2 n s_{13} .
$$

When $k$ and $n$ range over all integers, the set $G$ is a principal ideal of $\mathbf{Z}$. Hence we can choose $s^{\prime}$ as asserted. (2) is obvious.

REMARK 1. The set $k^{2} \cdot s_{13}^{2} s_{2} /\left(s_{13}, s_{2}\right)^{2}$ is a multiple of $s_{13}$. If we let $\tilde{s}_{13}=$ $s_{13} /\left(s_{13}, s_{2}\right)$ and $\tilde{s}_{2}=s_{2} /\left(s_{13}, s_{2}\right)$, then we have

$$
l\left(s_{23}\right)= \begin{cases}2\left(s_{23} \tilde{s}_{13}, s_{13}\right) & \text { if } \tilde{s}_{2} \text { is even and } s_{23} \neq 0 \\ \left(2 s_{23} \tilde{s}_{13}, s_{13}\right) & \text { if } \tilde{s}_{2} \text { is odd and } s_{23} \neq 0 \\ 2\left|s_{13}\right| & \text { if } s_{23}=0\end{cases}
$$


REMARK 2. The set $\delta\left(s_{13}, s_{2}\right)$ in Lemma 1 is a subset of $M\left(s_{13}, s_{2}\right)$ which consists of matrices in $\Delta\left(s_{13}, s_{2}\right)$ with $0 \leqslant s_{23}<\left(s_{13}, s_{2}\right)$ and $0 \leqslant s_{3}<2 s_{13}$. However, $\delta\left(s_{13}, s_{2}\right) \neq M\left(s_{13}, s_{2}\right)$ in general as shown by the following example:

$$
{ }^{t}\left[\begin{array}{ccc}
1 & -1 & -1 \\
0 & 1 & 1 \\
0 & 0 & 1
\end{array}\right]\left[\begin{array}{ccc}
0 & 0 & 3 \\
0 & 3 & 2 \\
3 & 2 & 1
\end{array}\right]\left[\begin{array}{ccc}
1 & -1 & -1 \\
0 & 1 & 1 \\
0 & 0 & 1
\end{array}\right]=\left[\begin{array}{lll}
0 & 0 & 3 \\
0 & 3 & 2 \\
3 & 2 & 2
\end{array}\right] .
$$

From this lemma, it is easy to see that $\mu\left(s_{13}, s_{2}\right)=\mu\left(s_{13},-s_{2}\right)=\mu\left(-s_{13}, s_{2}\right)=$ $\mu\left(-s_{13},-s_{2}\right)$. Hence it suffices to consider the case when $s_{13}$ and $s_{2}$ are positive integers. Here are some particular values of $\mu\left(s_{13}, s_{2}\right)$ for $s_{13}=1,2,3,4,5$.

$$
\begin{aligned}
& \mu\left(1, s_{2}\right)= \begin{cases}2 & \text { if } s_{2} \text { is even, } \\
1 & \text { if } s_{2} \text { is odd }\end{cases} \\
& \mu\left(2, s_{2}\right)= \begin{cases}4 & \text { if } s_{2}=4 n+1,4 n+3, \\
6 & \text { if } s_{2}=4 n+2,4 n ;\end{cases} \\
& \mu\left(3, s_{2}\right)= \begin{cases}3 & \text { if } s_{2}=6 n+1,6 n+5, \\
6 & \text { if } s_{2}=6 n+2,6 n+4, \\
5 & \text { if } s_{2}=6 n+3, \\
10 & \text { if } s_{2}=6 n ;\end{cases} \\
& \mu\left(4, s_{2}\right)= \begin{cases}8 & \text { if } s_{2} \text { is odd, } \\
12 & \text { if } s_{2}=8 n+2,8 n+6, \\
16 & \text { otherwise; }\end{cases} \\
& \mu\left(5, s_{2}\right)= \begin{cases}5 & \text { if } s_{2}=10 n+1,10 n+3,10 n+7,10 n+9, \\
10 & \text { if } s_{2}=10 n+2,10 n+4,10 n+6,10 n+8, \\
9 & \text { if } s_{2}=10 n+5, \\
18 & \text { if } s_{2}=10 n .\end{cases}
\end{aligned}
$$

For each fixed positive integer $s_{13}$, we define

$$
\eta\left(s_{13}\right)=\sum_{s_{2} \neq 0} \frac{\mu\left(s_{13}, s_{2}\right)}{s_{13}^{4} s_{2}^{2}} \cdot \frac{1}{2 \zeta(2)} .
$$

LEMMA 2. For any positive integer $k$, we have

$$
\eta\left(2^{k}\right)=\frac{2^{k+1}}{2^{4 k}}+\frac{2^{k}}{2^{4 k+2}}+\cdots+\frac{2^{k}}{2^{6 k}} .
$$

Proof. Since the values of $\mu\left(s_{13}, s_{2}\right)$ are computed via Lemma 1 as

it follows that

$$
\mu\left(2^{k}, s_{2}\right)= \begin{cases}2^{k+1}+m \cdot 2^{k} & \text { if }\left(s_{2}, 2^{k+1}\right)=2^{m}, 0 \leqslant m \leqslant k, \\ 2^{k+1}+k \cdot 2^{k} & \text { if }\left(s_{2}, 2^{k+1}\right)=2^{k+1}\end{cases}
$$

$$
\begin{aligned}
\eta\left(2^{k}\right) & =\sum_{s_{2}=1}^{\infty} \frac{\mu\left(2^{k}, s_{2}\right)}{2^{4 k} s_{2}^{2}} \cdot \frac{1}{\zeta(2)} \\
& =\frac{2^{k+1}}{2^{4 k}}+\frac{2^{k}}{2^{4 k+2}}+\cdots+\frac{2^{k}}{2^{6 k}}
\end{aligned}
$$


LEMMA 3. If $p$ is an odd prime and $m$ is a positive integer, then

$$
\eta\left(p^{m}\right)=\frac{5}{4}\left[\frac{p^{m}}{p^{4 m}}+\frac{\phi\left(p^{m}\right)}{p^{4 m+2}}+\cdots+\frac{\phi\left(p^{m}\right)}{p^{6 m}}\right],
$$

where $\phi\left(p^{m}\right)=p^{m-1}(p-1)$ is the Euler $\phi$-function.

Proof. Let $1 \leqslant n \leqslant m$ and $\left(s_{2}, 2 p^{m}\right)=2 p^{n}$. If $l\left(s_{23}\right)$ is the integer defined in Lemma 1 for such $s_{2}$, then we have $l(0)=2 p^{m}$ and $l\left(k p^{u}\right)=2 p^{m-n+u}$ if $(k, p)=1$ and $u$ is a nonnegative integer. (The total numbers of such $k$ 's is $\phi\left(p^{n-u}\right)$.) Hence we get

$$
\mu\left(p^{m}, s_{2}\right)=2\left[p^{m}+n \phi\left(p^{m}\right)\right] .
$$

For the case $\left(s_{2}, 2 p^{m}\right)=p^{n}$, we get $\mu\left(p^{m}, s_{2}\right)=p^{m}+n \phi\left(p^{m}\right)$ in the same manner. Hence our lemma follows from the definition of $\eta\left(p^{m}\right)$.

THEOREM B.

$$
\xi(2)=\frac{65}{24} \cdot \frac{\zeta(2) \zeta(3) \zeta(5)}{\zeta(6)}
$$

Proof. By the definition of $\eta$ and Lemma 1, we have

$$
\eta(a)=\alpha \sum_{d \mid a} \frac{a \phi(d)}{a^{4} d^{3}}
$$

where $\alpha=2$ if $a$ is even and $\alpha=\frac{5}{4}$ if $a$ is odd. Consequently, if $m$ and $n$ are relatively prime integers, then a direct calculation shows $\eta(m n)=\frac{4}{5} \eta(m) \eta(n)$.

Let $\tilde{\eta}(a)=\frac{4}{5} \eta(a)$. Then by the previous lemmas, we have the following properties for $\tilde{\eta}(m)$ :

$$
\begin{aligned}
& \tilde{\eta}\left(2^{k}\right)=\frac{8}{5}\left[\frac{2^{k}}{2^{4 k}}+\frac{2^{k-1}}{2^{4 k+2}}+\cdots+\frac{2^{k-1}}{2^{6 k}}\right] \\
& \tilde{\eta}\left(p^{k}\right)=\frac{p^{k}}{p^{4 k}}+\frac{\phi\left(p^{k}\right)}{p^{4 k+2}}+\cdots+\frac{\phi\left(p^{k}\right)}{p^{6 k}}
\end{aligned}
$$

if $p$ is an odd prime,

(3) $\tilde{\eta}(m n)=\tilde{\eta}(m) \tilde{\eta}(n)$ if $m$ and $n$ are relative prime integers.

Hence

$$
\sum_{m=1}^{\infty} \tilde{\eta}(m)=\prod_{p: \text { prime }}\left(1+\tilde{\eta}(p)+\tilde{\eta}\left(p^{2}\right)+\cdots+\tilde{\eta}\left(p^{n}\right)+\cdots\right) .
$$

For odd prime $p$, we have

$$
1+\sum_{k=1}^{\infty} \tilde{\eta}\left(p^{k}\right)=\frac{\left(1-p^{-6}\right)}{\left(1-p^{-3}\right)\left(1-p^{-5}\right)}
$$

For the special case $p=2$, we have

$$
1+\sum_{k=1}^{\infty} \tilde{\eta}\left(2^{k}\right)=\frac{13}{12} \cdot \frac{\left(1-2^{-6}\right)}{\left(1-2^{-3}\right)\left(1-2^{-5}\right)}
$$


Hence

$$
\begin{aligned}
\xi(2) & =2 \zeta(2) \cdot \sum_{m=1}^{\infty} \eta(m)=\frac{5}{2} \zeta(2) \sum_{m=1}^{\infty} \tilde{\eta}(m) \\
& =\frac{65}{24} \zeta(2) \prod_{p: \text { prime }} \frac{\left(1-p^{-6}\right)}{\left(1-p^{-3}\right)\left(1-p^{-5}\right)} \\
& =\frac{65}{24} \cdot \frac{\zeta(2) \zeta(3) \zeta(5)}{\zeta(6)} .
\end{aligned}
$$

3. The general case. For each fixed positive integer $s_{13}$, we define

$$
\eta_{t}\left(s_{13}\right)=\frac{1}{2 \zeta(2 t)} \cdot \sum_{s_{2} \neq 0} \frac{\mu\left(s_{13}, s_{2}\right)}{\left(s_{13}^{4} s_{2}^{2}\right)^{t}}, \quad \operatorname{Re} t \geqslant 1 .
$$

Then we have

(1) $\eta_{t}(1)=\frac{5}{4}$

$$
\begin{gathered}
\eta_{t}\left(2^{k}\right)=2\left(\frac{2^{k}}{2^{2 k t}}+\frac{2^{k-1}}{2^{(2 k+2) t}}+\cdots+\frac{2^{k-1}}{2^{3 k t}}\right), \\
\eta_{t}\left(p^{k}\right)=\frac{5}{4}\left(\frac{p^{k}}{p^{2 k t}}+\frac{\phi\left(p^{k}\right)}{p^{(2 k+2) t}}+\cdots+\frac{\phi\left(p^{k}\right)}{p^{3 k t}}\right)
\end{gathered}
$$

if $p$ is an odd prime,

(4) $\eta_{t}(m n)=\frac{4}{5} \eta_{t}(m) \eta_{t}(n)$ if $m$ and $n$ are relative prime integers.

From the computation we carried out before, we get

TheOREM A. For $\operatorname{Re} t \geqslant 2$, we have

$$
\xi(t)=\left[\frac{5}{2}+\frac{3}{2} \cdot \frac{2^{-2 t+1}+2^{-3 t}-2^{-5 t+2}}{1-2^{-3 t}}\right] \cdot \frac{\zeta(t) \zeta(2 t-1) \zeta(3 t-1)}{\zeta(3 t)} .
$$

4. Application and remark. Let $S$ be a $3 \times 3$ integral symmetric matrix of rank 3 . We call $S$ a zero ternary form if $S$ represents zero in rational integers; i.e. there exists a nonzero integral vector $u=\left[u_{1}, u_{2}, u_{3}\right]$ such that $u S u^{t}=0$. Hence there exists a unimodular integral matrix $U$ such that [3]

$$
U S U^{t}=\left[\begin{array}{ccc}
0 & 0 & s_{13} \\
0 & s_{2} & s_{23} \\
s_{13} & s_{23} & s_{3}
\end{array}\right] .
$$

Set

$\mathscr{A}$ : the set of representatives of zero ternary forms under the operation of unimodular matrices of $\mathrm{GL}_{3}(\mathbf{Z})$ by the action $S \rightarrow$ $U S U^{t}$,

$\mathscr{B}$ : the set of representatives of

$$
G=\bigcup_{s_{2} \neq 0} \bigcup_{s_{13}=1}^{\infty} \Delta\left(s_{13}, s_{2}\right)
$$

under the operation of $\mathscr{P}$. 
Then for each $S \in \mathscr{A}$, there exists a unimodular $U$ in $\mathrm{GL}_{3}(\mathbf{Z})$ such that $U S U^{t} \in \mathscr{B}$. Hence we can approximate the series

$$
\tilde{\xi}(t)=\sum_{x \in \mathscr{A}} \frac{1}{|\operatorname{det} x|^{t}}, \quad \operatorname{Re} t \geqslant 2,
$$

by the series

$$
\begin{aligned}
\xi(t) & =\sum_{x \in \mathscr{B}} \frac{1}{|\operatorname{det} x|^{t}} \quad(\operatorname{Re} t \geqslant 2) \\
& =\sum_{s_{2} \neq 0} \sum_{s_{13}=1}^{\infty} \frac{\mu\left(s_{13}, s_{2}\right)}{\left(s_{13}^{2} s_{2}\right)^{t}}
\end{aligned}
$$

which contains $\tilde{\xi}(t)$ as a subseries. If we use the approximate values of zeta-functions as

$$
\begin{array}{ll}
\zeta(2)=1.6449341, & \zeta(3)=1.2020569, \\
\zeta(5)=1.0369297, & \zeta(6)=1.0173431
\end{array}
$$

it follows that

$$
\frac{65}{48} \cdot \frac{\zeta(3) \zeta(5)}{\zeta(6)} \simeq 1.0086268 \zeta(2) .
$$

Hence it is possible that $\tilde{\xi}(2)=2 \zeta(2) \zeta(2)$ (a formula which is hard to verify directly).

Note that the zeta-function $\tilde{\xi}(t)$ we defined here is a constant multiple of a subseries of $\xi_{2}(s, L)$ appearing in [2] (restricted $L$ to zero ternary forms). This tells us that a constant multiple (the constant is $2^{-6} \pi^{-4}$ by a direct computation from the Selberg Trace Formula) of $\tilde{\xi}(2)$ gives the contribution of ternary forms to the dimension formula of Siegel's cusp forms of degree three with respect to $\operatorname{Sp}(3, \mathbf{Z})$.

\section{REFERENCES}

1. T. Shintani, Zeta-functions associated with the vector space, J. Fac. Sci. Univ. Tokyo Sect. 1A Math. 22 (1975), 25-65.

2. C. L. Siegel, Lectures on quadratic forms, Tata Inst. of Fundamental Research, Bombay, 1957.

3. , Über die Zetafunktionen indefiniter quadratischer Formen, Math. Z. 43 (1938), 682-708.

institute of Mathematics, Academia Sinica, Nankang, Taipei, Taiwan, Republic of China

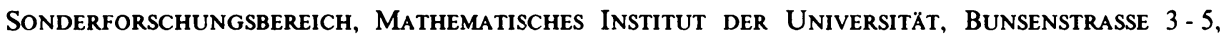
D - 3400 Gottingen, Federal Republic of Germany (Current address) 